\title{
Ciudades y Regiones frente a las transformaciones globales
}

\section{María del Huerto Romero *}

Óscar Madoery **

\section{Introducción}

A finales del siglo XX, el marco de actuación de las ciudades reconoce la intèrsección de tres dinámicas:

- Una nueva relación Estado-Nacionalterritorio subnacional, donde este último (en cualquiera de sus niveles: región, provincia o municipio) se constituye en el nuevo actor del desarrollo.

- Un avance en los procesos de integración regional, que hace que las políticas de planeamiento y desarrollo territorial deban estar diseñadas e implementadas en términos del amplio contexto del espacio integrado.

- Un mundo global, generador de una nueva "geografía del poder donde la competitividad se constituye en un objetivo claramente vinculado a cuestiones de orden territorial; y donde el incremento de la competencia se da entre estos territorios, y especialmente entre sus puntos nodales o centros - las ciudades-.

A partir del escenario estratégico para el desarrollo urbano construido por estas tres dinámicas, el propósito del presente trabajo consiste en dar cuenta de algunos de los desafíos de transformación del patrón de gestión local/regional generados por $\mathrm{cl}$ nuevo marco de actuación.

\section{Las ciudades en la economía global}

En las tres décadas posteriores a la II Guerra Mundial, la internacionalización económica tendió a fortalecer un sistema in- ternacional interestatal. Los Estados nacionales, líderes en los sectores económicos relevantes -especialmente las manufacturas y la extracción de materias primas - se constituian en los sujetos centrales de los regímenes de comercio internacional (SASSEN, 1997).

A partir de los ' 80 emerge una situación en el mundo basada en nuevos equilibrios y en nuevos flujos. Las tendencias uglobalizadoras", unidas a los cambios políticos mundiales (unipolaridad política y multipolaridad económica y comercial) y a la megatendencia "descentralizadora" (revolución científica y tecnológica, reforma del Estado, demandas autonómicas de la sociedad civil, privatización y desregulación) (BoISIER, 1991); generan una nueva geografia del poder. En ella, el "lugar/territorio" adquiere centralidad para los múltiples circuitos a través de los cuales la globalización económica se constituye. La economía global se materializa en una red transfronteriza de regiones estratégicas; y más específicamente, de sus centros: las ciudades estratégicas.

Ello se debe a la interacción de dos procesos (SASSEN, 1997):

- El aumentado en la escala y la complejidad de las transacciones económicas producido por el rápido crecimiento de la globalización: las empresas que operan globalmente necesitan comprar insumos cada vez más especializados, demandan concentración de infraestructura y comunica- cinnes de punta, y mercados de recursos humanos altaniente capacitados.

- La creciente intensidad de los servicios en la organización de la economía. Las grandes empresas globales necesitan acceso inmediato a empresas especializadas de servicios legales, contables, de gerenciamiento, etc. Las grandes firmas que compran esos servicios no necesitan establecer su sede en las grandes ciudades - aunque todavía prefieren hacerlo- pero requieren de un acceso a una red concen- 
trada de empresas de servicios localizadas en las ciudades.

Por lo general, son las grandes ciudades o las áreas metropolitanas los mejores productores de los insumos y servicios especializados requeridos por estos fenómenos.

En virtud de estos procesos, algunas ciudades vieron crecer desde los años 80 su concentración de poder económico. Son las denominadas ciudades globales: grandes urbes con una creciente participación como nuevos actores de las relaciones culturales, sociales, económicas y financieras a nivel mundial. Muchas de ellas operan, incluso, como lugares de coordinación, control y servicio para el capital global.

El novedoso sistema urbano transnacional se completa con las llamadas Ciudades de Dimensión Medias: ciudades estratégicas que, sin reunir las condiciones de "globales", acreditan ciertos elementos de competitividad que la hacen partícipe de los circuitos económicos globales (BoRJA, 1997a).

Este tejido constituye una nueva geografía económica de centralidad que atraviesa las fronteras nacionales y la división Norte-Sur, y señala la existencia de un proceso económico global con múltiples locaciones en más de un país.

\section{Las ciudades en los procesos de integración regional}

Los procesos de integración regional -al igual que las tendencias globalizadoras- modifican el marco en el cual se desarrollan las políticas urbanas.

Es en el ámbito local donde los ciudadanos sienten directa y cotidianamente las repercusiones (en términos de oportunidades y amenazas) de dichos procesos. Es por ello que los municipios no pueden ni deben satisfacerse más con una visión político-administrativa limitada a sus fronteras locales o nacionales.

En el esquema de integración más acabado hasta el presente - la Unión Europea - se percibe desde hace varios años un incremento de la importancia de las ciudades, como elementos centrales en el desarrollo de las regiones nacionales, los países y el bloque comunitario como un todo.

Tanto las ciudades como los organismos comunitarios reconocen dos principios en la construcción del espacio regional europeo (EuROCITTES, 1995):
I. Las ciudades deben ser vistas como los puntos focales del desarrollo y, como consecuencia de ello;

II. las políticas de planeamiento territorial y desarrollo de las ciudades deben ser diseñadas e implementadas no sólo en términos locales, sino en el amplio contexto del espacio comunitario.

Esto ha llevado a que la Unión Europea - si bien carece de mandato sobre políticas urbanas- haya comenzado a otorgar gran relevancia en sus programas a las cuestiones urbanas, a las relaciones entre ciudades y a su papel estratégico en la construcción comunitaria.

Sin embargo, las ciudades europeas reconocen que ello no es suficiente: se requiere el reconocimiento legal de la dimensión urbana del proceso comunitario. Un primer avance en este sentido fue la inclusión en el texto del Tratado de la Unión del "Principio de la Subsidiariedad o Proximidad y la creación del "Comité de las Regiones y los Poderes Locales.".

\section{La ciudad y el Estado-Nación}

El nuevo rol internacional de las ciudades, como no podría ser de otra manera, se produce en el marco de un replanteo global de la noción y las funciones del Estado-Nación y de las unidades territoriales subnacionales.

La internacionalización del flujo de decisiones estratégicas, como componente del nuevo escenario global, requiere por parte de los gobiernos capacidad de adaptación a realidades dinámicas, cambiantes e imprevisibles. Estamos ante un nuevo tiempo histórico donde la escena internacional pasa a ser el lugar donde se plasman los diferentes estilos nacionales de funcionamiento económico. Cada vez puede hablarse menos de economías nacionales, en su acepción tradicional, sino de espacios políticos nacionales operando en una trama indisoluble de procesos económicos mundiales (Dos Santos y CaLderón, 1993).

Esta situación obliga a los Estados nacionales a introducir reformas que optimicen sus estilos de intervención (reactivando mercados, reorganizando sus estructuras de gestión, acentuando la calidad de las interacciones de flujos y comunicaciones, horizontalizando y descentralizando procesos decisorios), en pos de una mayor competitividad internacional.

En el caso de los países latinoamericanos, y en particular de la Argentina, habría que agregar otro elemento a esta situación: 
el doble desbordamiento del Estado, con pérdida de soberanías instrumentales hacia ámbitos económicos políticos integrados como el Mercosur, y con una crisis de gestión pública hacia adentro, que se manifiesta en la incapacidad de administrar y asignar recursos en forma eficiente y equitativa.

La aparición de nuevos agentes privados y públicos modifican aspectos básicos de los mecanismos de intervención estatal, ayudando a configurar un marco de redefinición de roles y de nuevos encuentros público-privado. De este modo, la reforma del Estado es un proceso que actualiza el problema de la eficiencia, la equidad y el protagonismo social en la gestión pública y, consecuentemente, replantea la problemática de la redistribución territorial de responsabilidades en la formulación e implementación de las políticas públicas, para impulsar mejor aquellos objetivos.

El efecto combinado de estos fenómenos es la fragmentación de las trayectorias del desarrollo. Las regiones y ciudades, ya no tienen un horizonte prefijado, ni establecido centralmente, sino que deben ser gestores de su propio desarrollo. Estrategias de desarrollo pensadas desde lo local y regional, aparecen como una respuesta innovadora a los problemas de crecimiento, competitividad y generación de empleo estable y con futuro.

La ciudad cambia su rol de mera proveedora de servicios públicos o ejecutora local de decisiones nacionales, y pasa a ser un laboratorio privilegiado para poner en escena un nuevo modelo que complementa $-\mathrm{y}$ no sustituye - a los anteriores (Díaz, 1994).

El nuevo patrón de gestión local debe contemplar:

I. El nuevo marco de actuación dado por los proceso de globalización y regionalización.

II. La ampliación de la esfera de actuación de los gobiemos locales, agregando a sus funciones tradicionales (obra pública, provisión de servicios básicos, regulación de la vida comunitaria) el diseño e implementación de estrategias de desarrollo local tendentes a proyectar externamente la ciudad y generar ventajas competitivas territoriales.

Hemos planteado hasta aquí el marco de actuación de las políticas urbanas. En él, el desafío más importante para todo gobierno territorial (sea a nivel de región, provincia o ciudad) es cómo ayudar a su propio territorio a posicionarse de una manera moderna y competitiva en el contexto internacional y, al mismo tiempo, de modo equitativo y participativo en el marco interno (BOISIER, 1996).
Enfrentar dicho desafío implica pensar y actuar en función de dos dinámicas propias del nuevo escenario estratégico para el desarrollo urbano:

$\Rightarrow$ la competitividaud iniemacionrâl de lâs regiones/ciudades y las nuevas modalidades de gestión;

$\Rightarrow$ las nuevas modalidades de organización urbano-regionales.

\section{La competitividad internacionales de las ciudades y regiones}

En el actual mundo global la competitividad se constituye en un objetivo claramente vinculado a cuestiones de orden territorial: la creación de ventajas competitivas se logra mediante un proceso altamente localizado, territorialmente definido.

$\mathrm{Al}$ "construirse" una región o un territoriọ relevante desde el punto de vista de la competitividad, el espacio se convierte en una variable estratégica de la competitividad de las empresas y organizaciones.

Estos atributos hacen referencia a un concepto sistémico de competitividad: son los sistemas de relaciones productivas, tecnológicas, comerciales y de servicios qùe se estructuran alrededor de un producto competitivo los que le otorgan tal caracter, más allá de la productividad del proceso mismo. La red de subcontratistas, transportistas, prestadores de servicios y proveedores en torno de una producción competitiva se configura territorialmente y demuestra una alta densidad de interacciones en el espacio geográfico próximo a la empresa, ámbito que coincide con un territorio local o regional (BoISIER, 1996)

El desarrollo territorial constituye, de este modo, un fenómeno de tipo empresarial e intensamente localizado, centrado en las ciudades donde las empresas sitúan sus unidades organizativas de negocio y en el sistema de ciudades que da soporte al flujo de relaciones internacionales de cada sistema productivo local y regional. "En el mercado internacional ya no existen empresas aisladas que compitan en forma descentralizada; se constituyen agrupamientos industriales de empresas organizados en redes cuya dinámica de desarrollo depende esencialmente de la eficacia de su localización industrial...." (MFssnfr., 1996).

En síntesis, dentro del marco de fuertes transformaciones que se dan en las relaciones políticas, económicas y humanas, resulta cada vez más evidente que existe una estrecha interrelación entre los procesos de crecimiento económico y de desarro- 
llo territorial, y entre competitividad de las organizaciones empresariales y la de los territorios donde éstas se localizan (CотоRRUELO MENTA, 1995).

Jordi BoRJA (1997) plantea los elementos centrales de los cuales dependerá la competitividad de los territorios:

$\Rightarrow$ Un funcionamiento eficiente del sistema urbano-regional, especialmente en cuanto a movilidad y dotación infraestructural (accesibilidad, suelo industrial, espacio de oficinas, etc.); y servicios básicos.

$\Rightarrow$ Su inserción en los sistemas de comunicaciones de carácter global y buena información de los agentes sociales y económicos de los procesos mundiales.

$\Rightarrow$ Cualificación de los recursos humanos (no únicamente de los estratos superiores) y capacidad de innovación (formación profesional y modernización tecnológica y empresarial).

$\Rightarrow$ Un apoyo público a los agentes económicos y sociales por medio de políticas de proteccionismo exportador, favoreciendo las sinergias y la innovación continuada.

$\Rightarrow$ Instituciones públicas representativas, eficaces y transparentes que actúen según normas claras y estables en sus relaciones con los agentes privados.

$\Rightarrow$ La definición de un proyecto de ciudad (o región) y marketing del mismo.

$\Rightarrow$ Una gobernabilidad del territorio basada en la cohesión y la participación cívica.

$\Rightarrow$ La calidad ambiental y social (localización, vivienda, clima social, cultural, etc.).

La gestión estratégica del desarrollo reclama, entonces, que las ciudades/regiones se conciban como una organización que produce y gestiona bienes y servicios y compite con otras ciudades/regiones en los mercados nacionales e internacionales (Cotorruelo Menta y otros, 1993).

Sin embargo, para evitar construir una ciudad que si bien sea atractiva hacia el exterior, profundice la geografía de centralidad y marginalidad en su interior, la ciudad debe incorporar políticas que promuevan y aseguren territorialmente el desarrollo competitivo (eficiencia), equitativo (equidad) y sostenible (equilibrio medioambental). Si el aumento de productividad y competitividad de la economía en su conjunto no se realiza con equidad y participación y sobre bases ambientalmente sustentables, el modelo termina siendo inviable a mediano plazo, en condiciones de gobernabilidad democrática.

Si bien la competencia entre territorios es una de las fuerzas motrices del actual mundo global, el escenario estratégico para el desarrollo urbano exige también formas de cooperación entre las ciudades que permitan operar en marcos más amplios. El bino- mio cooperación-competencia es uno de los elementos que marcan las relaciones en el sistema urbano mundial. La cooperación, necesaria para insertarse en un sistema internacional, tiene como objetivo último la mejora de la competitividad. Se coopera para poder competir mejor" (BORJA, 1997a).

Este nuevo patrón de gestión estratégica nos permite avanzar hacia una visión más enriquecedora del desarrollo: aquella que expresa que la ciudad no está (no puede estarlo) definida por fronteras a priori, sino que es una categoría flexible, reconocida por dinámicas específicas que la identifican (CASAIET, 1996).

Ello implica para las ciudades trabajar sobre un nuevo concepto de "región" y adaptar la gestión a "nuevas modalidades de organización urbano-regional.

\section{Las nuevas modalidades de organización urbano-regional}

Los impactos de los procesos de globalización e integración regional, la revolución científico-técnica, la reducción de los costos de transporte y comunicaciones, producen cambios contextuales y estructurales en tomo a la idea de región. Aquellos conceptos tradicionalmente ligados a su definición -como la contigüidad geográfica - han entrado en una fase de obsolescencia.

Es por ello que resulta necesario interpretar la idea de "región" como una estructura flexible, cuyos límites no necesariamente deben ser fijados en términos jurisdiccionales- ya que de esta manera solamente se atendería a uno de sus elementos constitutivos- sino en función de un abanico de aspectos:

- Eslabonamiento de procesos productivos (vinculación de actividades productivas).

- Articulaciones sociales (sectores sociales predominantes, formas organizativas).

- Recursos que lo rodean (factores productivos predominantes).

- Emprendimientos comunes (obras, planes, instituciones).

- Problemáticas concretas (demandas sociales focalizadas, desafíos competitivos, negociaciones similares con otras instancias estatales, fuga de factores regionales de producción).

La flexibilidad, la elasticidad y la maleabilidad se convierten en los elementos indispensables de toda región moderna.

-Actualmente una región es una estructura compleja e interactiva, con múltiples acotamientos, en la cual el conteni- 
do define el continente (limites, dimensiones y otros atributos geográficos). Hoy una región es una y múltiple simultáneamente, puesto que ya supera la noción de contigüidad, cualquiera de ella puede establecer alianzas tácticas con otras regiones para lograr objetivos específicos y por plazo determinados, a fin de posicionarse mejor en el contexto internacional. A partir de un núcleo original (que nunca deja de serlo) se configuran múltiples espirales asociativas que dan origen a nuevas instancias regionales, sin que la unidad básica pierda su propia naturaleza. (BoIsier, 1996).

Teniendo en cuenta estas características, el autor plantea tres modalidades de organización urbano-regional:

Regiones Pivotales: ‘territorios organizados, complejos e identificables a la escala de la división político-administrativa histórica. Estas regiones pivotales serán provincias en algunos países, departamentos en otros y estados en algunos paises federales. En todos los casos son las menores unidades político-administrativas que al mismo tiempo son estructuralmente complejas, poseen cultura e identidad y tienen flexibilidad.

Regiones Asociativas: "regiones de mayor amplitud a partir de la unión voluntaria de regiones pivotales con unidades territoriales adyacentes".

Regiones Virtuales: es el resultado de acuerdos cooperativos tácticos (formales o no) entre dos o más regiones pivotales o bien asociativas (no necesariamente contiguas o ni siquiera del mismo país) para alcanzar ciertos objetivos de corto y mediano plazo.

En un primer análisis pareciera que BoIsIER no incluye a las ciudades como regiones pivotales: "serán las menores unidades político-administrativas que al mismo tiempo son estructuralmente complejas, poseen cultura e identidad y tienen flexibilidad, y agrega, "serán provincias en algunos países, departamentos en otros y estados en algunos países federales".

Sin embargo, una interpretación más profunda de su propuesta, podría llegar a incluir a las grandes urbes. Al enunciar el autor dos ejemplos de regiones virtuales (cuyos núcleos son las regiones pivotales, como tales o asociadas) menciona a alos protocolos de cooperación de regiones y municipios europeos con otras regiones y municipios comunitarios para el desarrollo de programas conjuntos . En particular, hace referencia en Bélgica al programa LEDA (Liège-Europe Development Action) que tiene el propósito de impulsar y reforzar las alianzas entre Lieja y otras ciudades comparables en el plano internacional.

Para enfrentar el desafio de posicionar a su propio territorio de una manera moderna y competitiva en el contexto internacional, las ciudades deben incorporar a su patrón de gestión local las nuevas modalidades de organización urbano-regionales.
Esto es: abordar una dimensión metropolitana y organizarse como espacios (dentro o fuera de una misma nación) interconectados en red.

\section{Áreas Metropolitanas y Regiones Asociativas}

La «internacionalización de las ciudades se ve acompañada por otro fenómeno común tanto en las economías más desarrolladas como en las del Tercer Mundo: el crecimiento de las grandes urbes y sus áreas metropolitanas. Este fenómeno lleva implícito la necesidad de una nueva organización del territorio y de su gestión.

Frente a las condiciones y desafíos impuestos por la globalización, la concentración de actividades y funciones, y la comunicación, las grandes ciudades deben trascender sus límites jurisdiccionales estrictos y abordar una dimensión mayor a escala metropolitana. La atención de las cuestiones metropolitanas no puede ser enfocada desde los simples ámbitos locales que constituyen pequeñas partes de un conjunto. Requiere visiones globales y tratamientos unificados.

Habitualmente en una estructura metropolitana se presenta una unidad dominante ( ala ciudad centrab) y, por otro lado, una extensión metropolitana de mayor o menor dimensión (subsistema policéntrico). Tradicionalmente se ha concebido a este sistema metropolitano como "territorialmente definido y estable, interactivo, jerárquico y aún solidario" (Subsecretaría de Planeamiento y Control de Gestión de la Provincia de Santa Fe, 1993). Esta delimitación hoy es superada por otras visiones más comprensivas del fenómeno, que consideran inconveniente limitarlo a un área geográfica rígida. Se refuerza la idea de funcionalidad (identificar problemáticas metropolitanas y desarrollar acciones metropolitanas para cada caso detectado) por sobre la de delimitación espacial.

En el marco de las nuevas modalidades de organización urbano-regionales, un área metropolitana (en su acepción más moderna) respondería, entonces, al modelo de "región asociativa*; donde:

- la región pivotal estaría constituida por la ciudad central, a la escala de la división político-administrativa;

- la región asociativa estaría constituida a partir de la unión voluntaria de la región pivotal con unidades territoriales adyacentes (bien del Área Metropolitana, bien de la Región Metropolitana) para desarrollar Acciones Metropolitanas en forma concertada y conjunta para cada problemática detectada; no siendo condición ne- 
cesaria el establecimiento legal de una perimetración rígida.

La nueva realidad metropolitana debe entenderse como un sistema o una red de geometría variable articulado por nodos, puntos fuertes de centralidad definidos" (BoRJA, 1997). En este marco, el trabajo en red se constituye en una herramienta fundamental para el funcionamiento de las regiones asociativas/espacio metropolitano.

\section{Redes de Ciudades y Regiones Virtuales}

La cooperación a partir del trabajo en red es para las ciudades un aspecto de su estrategia de inserción en el entorno internacional y una estrategia de mejora de su competitividad.

CamagnI define las redes de ciudades como asistemas de relaciones y de flujos, de carácter preferentemente horizontal y no jerárquico, que se establecen entre centros complementarios o similares y que garantizan la creación de externalidades o economías de especialización, complementariedad, división espacial del trabajo, de cooperación, sinergia e innovación" (citado por VázQuez Barquero, 1997).

Las redes de ciudades han sido clasificadas de muy diversas maneras. En términos generales, es posible identificarlas básicamente en tres grandes grupos (BoRJA, 1997b):

Las redes sectoriales pueden jugar un papel importante en la mejora de los intercambios y las infraestructuras de las ciudades. Incluye las redes de puertos, de ferias, de centros de distribución, etc. También se incluyen aquellas redes sectoriales ligadas a la producción o los servicios y ofertas comunes.

Las redes globales son redes de cooperación y competencia entre iguales, por ejemplo EuRocimes. Sirven para la cooperación y la elaboración de estrategias comunes, además de ser redes de representatividad y lobby político.

Las redes de coordinación o de aglomeración -redes regionales o macrorregionales-, son redes con un mayor impacto territorial ya que constituyen un nuevo espacio regional de actuación. Este tipo de redes se forma para la coordinación de grandes proyectos o de planes estratégicos que proyectan el nuevo espacio regional, constituido por las ciudadés principales de la red. Se orientan a la mejora de la competitividad de sus miembros a partir de las economías de aglomeración y de escala. El caso de la zona metropolitana de Lille es un ejemplo de ello. Son las que tienen un ma- yor impacto sobre la organización territorial —por ejemplo en la Unión Europea - ya que cambian el peso específico de áreas concretas.

En el apartado anterior, hemos señalado que el trabajo en red puede constituir una modalidad de organización entre ciudades para la conformación de áreas metropolitanas o regiones asociativas con el objeto de encarar problemáticas comunes. Tal el caso de Bilbao Metrópoli 30.

Sin embargo, la creciente interdependencia y complementariedad del territorio organizado ha llevado a que en los últimos años ciudades distantes (dentro o fuera de una misma nación) tiendan también a organizarse como espacios interconectados en red. Este tipo de redes de ciudades dan lugar a la formación de la nueva modalidad de organización urbano-regional: la aregión virtuab.

Ella encuentra su respaldo teórico en la propuesta cepalina de regionalismo abierto para los países (esto es, la posibilidad que tiene cualquier país de pertenecer simultáneamente a varios esquemas de integración), y, más específicamente, en la adaptación hecha por el ILPES para los territorios subnacionales: el regionalismo virtual:

- El regionalismo virtual surge como un instrumento para la cooperación entre territorios onganizados para la competencia y competitividad internacional .... en un escenario mundial caracterizado por la globalización y la reestructuración económica y politica subnacional.

Las principales caracteristicas del regionalismo virtual son: flexibilidad, elasticidad y colapsibilidad. La continuidad geográfica y el tamaño pasan a ser reemplazados por relaciones funcionales expresadas en el espacio decisional y por el nivel de complejidad del territorio, permitiendo la asociatividad y el virtualismo sin perdida de identidad corporativa (LIRA, 1995)

En los últimos años, las redes transfronterizas entre ciudades han ido adquiriendo una importancia creciente, particularmente como ejes de desarrollo en los procesos de integración regional. Ejemplo de ello son las redes europeas de ciudades, como Eurocities.

Algunas ciudades latinoamericanas también han definido su voluntad de trabajo concertado y de participación en la construcción de los procesos de integración regional. Sin el alcance asociativo ni la multiplicidad de redes que existen en Europa, América Latina ha comenzado a trabajar sobre la constitución de aregiones virtualess. En algunos casos se trata de proyectos iniciales (por ejemplo: propuestas de coordinación entre ciudades destinadas a constituir ejes o sistemas urbanos, como el eje de las ciudades del corredor bioceánico), y en otros, de iniciativas que han alzanzado mayor nivel de avance (como la Red de Mercociudades). 
- Docente-investigadora de la Facultad de Ciencia Política y Relaciones Intemacionales de la Universidad Nacional de Rosario (Argentina).

- Docente de la Facultad de Ciencia Política y Relaciones Intemacionales e investigador del CIUNR, Universidad Nacional de Rosario (Argentina).

Ambos autores formaron parte del equipo de investigación que brindó asistencia técnica al Plan Estratégico Rosario, en el marco del Convenio Municipalidad de Rosa-
rio/Facultad de Ciencia Política y Relaciones Intemacionales de la Universidad Nacional de Rosario. Integraron también dicho equipo: Dr.Arturo Fernández, Lic. Silvia Ga-

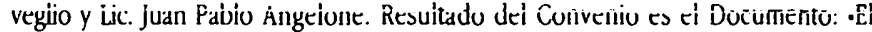
Rol de Rosario en un Sistema Regional de Ciudades. Rosario: Centro Geopolitico-económico y Puerta y Puerto del Mercosur, algunas de cuyas ideas se abordan en este articulo.

\section{Bibliografia}

BolsIER, Sergio (1991), -La descentralización: un tema difuso y confuso* en D. NOHLE: (comp.), Descentralización política y consolidación democrática. Europa-América del Sur, Editorial Nueva Sociedad, Caracas.

_._(1996), •Modemidad y Territorio, Cuademos del ILPES, n. 42, Santiago.

BORJA, Jordi (1997), ePlaneamiento estratégico y proyectos urbanos: nuevos territorios y nuevas economías, mimeo, Rosario.

(1997a), sLa internacionalización de las ciudades, en Revista Córdoba. Ciudad y Desarrollo, Año III, n. ${ }^{9}$, Córdoba, enero-junio.

__(1997b) .Ciudad, Competitividad e Integración. El rol de las ciudades en la construcción de Europa y la emergencia de políticas urbanas en la Unión Europea., III Reunión Cumbre de Ciudades del MERCOSUR, Córdoba, septiembre.

Caballero, Adrián (1997), ·Rosario Metropolitano•, Informe presentado al Plan Estratégico de Rosario; mimeo, Rosario

CASAIET, Mónica (1996), -Introducción, en -Redes y Regiones, una nueva configuración., Revista Latinoamericana de Estudios del Trabajo, Año 2, n.³, México.

Cotorruelo Menta, Romeo (1995), Planificación y gestión estratégica del desarrollo local, Inmark Argentina, Buenos Aires.

; Vázquez Barquero, Antonio; Riestra Cherbavaz, R. y Ursa, Y. (1993), -Bases para un modelo de desarrollo regional apoyado en el sistema de ciudades. Documento del Grupo Inmark, Madrid, abril.
Díaz, Antonio (1994), •Municipio Relacional: la Eficacia Social, en Nueva Gestión Local, Editorial Popular, Madrid.

Dos Santos, Mario y CALDERON, Femando (1993), • Hueso Duro, mimeo, Buenos Aires.

EuRocrines (1995), •Eurocities Workshop for Elected Members, Concluding Remarks of the Round Tables on: -Europe 2000 and -Cities and the Trans-european Networks, Bruselas, setiembre.

(1996), $A$ A Charter of the European Cities. Towards the Revision of the Treaty on European Union, Bruselas, marzo.

LIRA, Luis (1995), -Regionalismo abierto y regionalismo virtual., Documento 95/13, ILPES, Santiago.

MESSNER, Dirk (1996), •Dimensiones espaciales de la competitividad internacional., en -Redes y Regiones, una nueva configuración., Revista Latinoamericana de Estudios del Trabajo, Año 2, n.. 3, México.

SASSEN, Saskia (1997), :Cities in the Global Economy, Simposio -La Ciudad Latinoamericana y del Caribe en el Nuevo Siglo, BID, Barcelona, España, marzo.

Subsecretaría de Planeamiento y Control de Gestión de la provincia de Santa fe (1993), .Proyecto: Extensión Metropolitana de Rosario. Proceso de formación urbanoterritorial y dinámicas de transformación, Santa Fe.

VÁzQuez Barquero, Antonio (1997), PPrograma de Teoria Económica y Desarrollo Territorial, Curso brindado en la Universidad Católica de Montevideo, Unuguay. 
